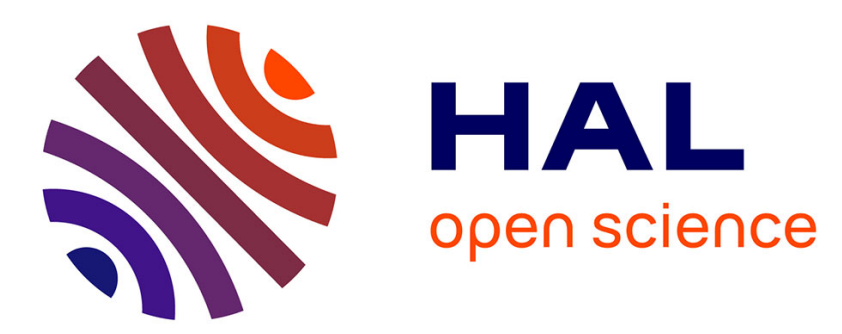

\title{
Simulation of Viscous Fingering in Rectangular Porous Media with Lateral Injection and Two- and Three-Phase Flows
}

\author{
Bertrand Lagrée, Stéphane Zaleski, Igor Bondino
}

\section{- To cite this version:}

Bertrand Lagrée, Stéphane Zaleski, Igor Bondino. Simulation of Viscous Fingering in Rectangular Porous Media with Lateral Injection and Two- and Three-Phase Flows. Transport in Porous Media, 2016, 113, pp.491 - 510. 10.1007/s11242-016-0707-x . hal-01424259

\section{HAL Id: hal-01424259 \\ https://hal.sorbonne-universite.fr/hal-01424259}

Submitted on 2 Jan 2017

HAL is a multi-disciplinary open access archive for the deposit and dissemination of scientific research documents, whether they are published or not. The documents may come from teaching and research institutions in France or abroad, or from public or private research centers.
L'archive ouverte pluridisciplinaire HAL, est destinée au dépôt et à la diffusion de documents scientifiques de niveau recherche, publiés ou non, émanant des établissements d'enseignement et de recherche français ou étrangers, des laboratoires publics ou privés. 


\title{
Simulation of viscous fingering in rectangular porous media with lateral injection and two- and three-phase flows
}

\author{
Bertrand Lagrée ${ }^{1,2,3}$, Stéphane Zaleski ${ }^{2,3}$, and Igor \\ Bondino $^{1}$ \\ ${ }^{1}$ TOTAL SA \\ ${ }^{2}$ Sorbonne Universités, UPMC Univ Paris 06, UMR \\ 7190, Institut Jean le Rond d'Alembert, F-75005, Paris, \\ France \\ ${ }^{3}$ CNRS, UMR 7190, Institut Jean le Rond d'Alembert, \\ F-75005, Paris, France
}

Received: date / Accepted: date

\begin{abstract}
Understanding multiphase flow in porous media is of tremendous importance for many industrial and environmental applications at various spatial and temporal scales. The present study consequently focuses on modeling multiphase flows by the Volume-of-Fluid method (sharp interface) in porous media with a simplified Darcy-scale approach and shows simulations of Saffman-Taylor fingering. The simplification of the Darcy scale approach is performed by assuming sharp interfaces between pure phases. The Volume-of-Fluid method with octree mesh refinement is used. It is implemented in the Gerris code which allows efficient parallel computations. We measure the scaling properties of the fractal viscousfingering patterns that appear in the numerical simulations. One of these properties is the fractal or Hausdorff dimension $D_{F}$. The other is the variation of the area $A$ of the viscousfingering cluster with the length $L$ of its perimeter, which varies as a simple power law $A \sim L^{\alpha}$. The injection of an intermediate-viscosity Newtonian fluid as a second step is also simulated. We are thus able to observe an increase of recovery of the high-viscosity fluid behind the fingering front, due to the reduction of the viscosity contrast. Some of these results are compared to waterflooding experiments of extra-heavy oils in quasi-2D square slab geometries of Bentheimer sandstone.
\end{abstract}

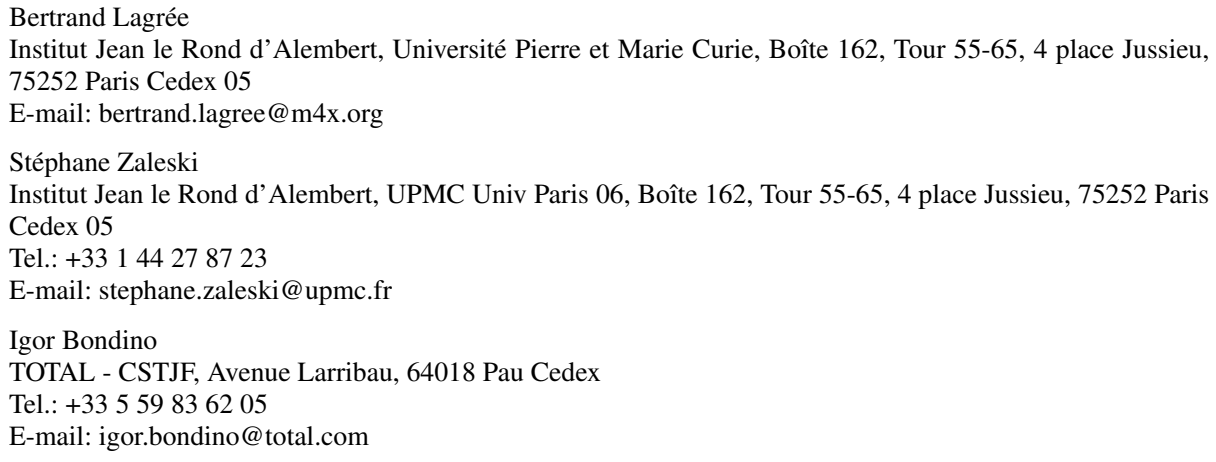


Keywords Volume-of-Fluid · Viscous fingering - Multiphase flows - Fractal patterns · Invasion at breakthrough

PACS 47.20.Cq 4 47.20.Ma $47.56 .+\mathrm{r}$

\section{Introduction}

When a porous solid matrix is fully saturated with one fluid phase, the penetration of another fluid phase displays fascinating physics. In this paper we consider this invasion problem numerically in rectangular slab of moderate thickness, with one or two invading fluids and with several drastic simplifying assumptions. A major simplifying assumption is to consider the flow at a scale (the Darcy scale) much larger than the pore scale. The main motivation for investigating the flow at this scale is the drastic simplification it allows, yielding a mathematical problem that is much more amenable to analysis. When the invading fluid is injected from one side of the slab, several physical effects cause the front to deviate from a flat shape. The heterogeneity of the porous media, that is the variable shape and size of the pores, and the spatial fluctuations of capillary forces make the interface rough. Moreover, when the invading fluid is less viscous than the receding fluid, the flat front is subject to an instability purely due to the viscosity difference. The instability is found theoretically in the small perturbation regime and is observed numerically and physically in a large perturbation regime in which it leads to complex branched structures. It is usually called Saffman and Taylor (1958) or viscous fingering instability.

In this paper we focus on the viscous fingering instability and neglect the other effects such as the heterogeneity of the medium or capillarity. In addition, we neglect the thickness of the front, that is we disregard the existence of a thick interface in which both phases coexist. Moroever we assume that on each side of the front a single phase is present. While these assumptions are not realistic, they allow to separate the various effects at play in the real physical phenomenom and to discover what is purely the effect of the viscosity contrast with a sharp front.

There is another interesting fact in favor of this simplifiation. There is a more complex models in which regions of mixed phase are considered, the multiscale Darcy equations. These have in 1D exact solutions, the Buckley-Leverett solution (Buckley and Leverett, 1942) that involves the formation of a sharp discontinuity in saturation and thus a sharp discontinuity in mobility. Thus the evolution of a sharply discontinuous interface is not completely irrelevant to the real-world physics with mixed phases at the Darcy scale.

The viscous fingering instability leads to complex structures which were most frequently observed in model experiments performed in Hele-Shaw (1898) cells rather than in porous media but are seen in both. These complex structures are similar to fractal sets and can be characterized by several exponents such as the fractal dimension. We think it is of interest to measure these exponents in simulations of our simplified model as in real experiments in order to understand how much of the real world structures can be explained by a simple viscous fingering model at the Darcy scale.

While observations and measurements of the fractal nature of invasion have frequently been performed in experiments both of Hele-Shaw type (Praud and Swinney, 2005) and in actual porous media (Måløy et al., 1985; Feder, 1988), almost no numerical simulations showing fractal structures, with or without the addition of surface tension, have been performed. Almost the single exception are the works of Fast and Shelley (2006); Li et al. (2007) on central injection but due to the high cost of the simulations only a moderately 


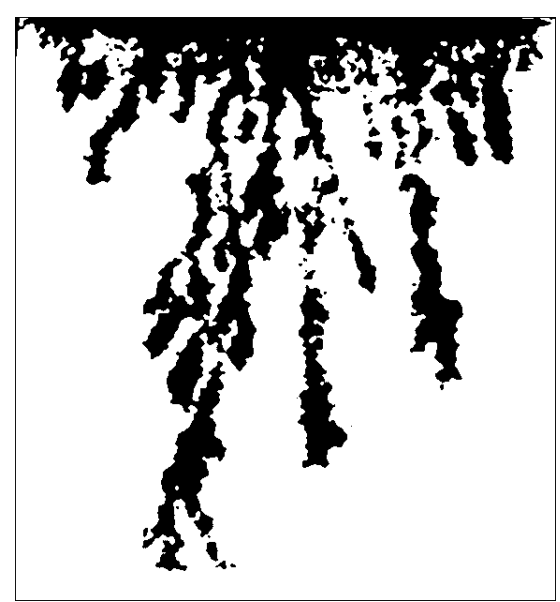

Fig. 1 2D imaging of waterflood in Bentheimer sandstone with $2.0 \mathrm{~kg} \cdot \mathrm{m}^{-1} \cdot \mathrm{s}^{-1}$ crude oil. Water is injected from the top, in black is the injected water. From Skauge et al. (2012) after applying a threshold to the image

wide range of scales has been seen in these cases. Because of the moderate range of scales an estimation of a fractal dimension $D_{F}$ would have been difficult in the already published cases, and the authors resorted instead to the measurement of the correlation between object area $A$ and object perimeter $L$ in the form $A \sim L^{\alpha}$. In recent work by us and other authors (Lagrée et al., 2014) both $D_{F}$ and $\alpha$ are measured for central injection but not for lateral injection, as we shall do below.

We compare the results of simulation in the frame of this model with an actual invasion experiment by Skauge et al. (2012) shown in Figure 1. This experiment has the advantage of being performed in a slab with square geometry with a moderate thickness so it matches approximately our model. However the slab thickness is such that the variation of the saturations in the transverse direction cannot be disregarded so we only expect a qualitative agreement with the model.

In the first part of the paper we consider the invasion of one fluid by another, then in a second part we consider the subsequent invasion by a third fluid, motivated by industrial processes where such a second invasion is performed. Our paper is organized as follows: in the second section we describe the simplified model and the instability, then in the third section the numerical method. In the fourth section we discuss results.

\section{Simplified viscous flow model}

We study the development of viscous fingering in a porous slab with lateral injection. The flow is supposed to be uniform in the transverse direction $z$ and attention is focused on a rectangular domain. This domain is initially filled with a Newtonian, high-viscosity fluid. A less viscous fluid is injected from one side of the domain with a constant velocity $U$. The two fluids then occupy two subdomains separated by a smooth, infinitely thin interface. The side boundaries $y= \pm L_{y} / 2$ are impermeable while the right boundary at $x=L_{x} / 2$ allows a constant pressure outflow condition for the fluids. 
We consider incompressible flow $(\nabla \cdot \mathbf{u}=0)$ that obeys Darcy's law

$$
\mathbf{u}=-\frac{k}{\mu} \nabla p
$$

with $k$ the permeability of the porous domain and $\mu$ the viscosity of the fluid. Two viscosities are considered $\mu_{a}$ for the advancing fluid and $\mu_{r}$ for the receding fluid.

In the case of simultaneous flow of two (or more) phases, boundary conditions must be given at the interface. Mass conservation imposes that

$$
[[\mathbf{n} \cdot \mathbf{u} \rrbracket=0
$$

Above $\left[[X]\right.$ denotes the jump $X_{2}-X_{1}$ of a variable $X$ accross the interface between subdomains 1 and 2 . When performing volume averaging, conserved quantities appear as flux terms on the surface of the volume and these jump conditions remain valid even for a thick front or at the Darcy scale. We disregard any effect of surface tension forces at the scale described by our model as discussed above so that the pressure jump due to capillary forces at the interface is constant

$$
\llbracket p \rrbracket=\frac{\sigma}{R_{p}}
$$

where $\sigma$ is the surface tension and $R_{p}$ is a constant having the dimension of a length. Redefining the pressure as

$$
p^{\prime}=p+\frac{\sigma}{R_{p}} H(\mathbf{x})
$$

where $H$ is the characteristic function of phase 2, so that $H=1$ in phase 2 and $H=1$ in phase 1 , the new pressure $p^{\prime}$ is continuous at the interface. Using the new, continuous pressure $p^{\prime}$ surface tension entirely disappears from the equations and Darcy's law (equation (1)) applies to the entire domain in the so called one-fluid approach (Tryggvason et al., 2011).

Dimensional analysis can be performed as follows. Considering the material properties and the flow variables, we have the permeability $k$ of the porous medium, the injection velocity $U$ and the viscosities of the fluids $\mu_{i}$. However, as the permeability $k$ and the viscosities $\mu_{i}$ only intervene as the ratio $k / \mu_{i}$, there is no intrinsic lengthscale in the idealized problem of a perturbed interface in an infinite domain although extrinsic lengths appear such as the simulation domain size. Similarly, the grid scale is invisible at the model scale. The grid spacing $h$ is thus the smallest resolved lengthscale, and as such the smallest lengthscale at which a fingering process can be observed (the small lengthscale of the fractal). As in more realistic models a flat interface is unstable with respect to small perturbations of wavenumber $q$. As shown by Chuoke et al. (1959) the growth rate $s$ is given by

$$
s=\frac{\mu_{r}-\mu_{a}}{\mu_{r}+\mu_{a}} U q
$$

where the index $r$ indicates the receding and $a$ the advancing fluid. In real experiments, the growth rate would be limited at large wavenumber $q$ by any surface tension effects. Wavelengths smaller than a critical length of order $(k \sigma / \mu U)^{1 / 2}$ will then not grow. In numerical simulations modes of wavelengths close to the grid size $h$ are also often damped. However, one may wonder about the relevance of adding a very small surface tension to our model in order to have a smoother of the interface at the grid scale. This was actually tried by Lagrée et al. (2014) but did not provide any significant changes in the observed shapes, so that we decided not to introduce surface tension in the current model. 
As a result complex structures grow and do not have any lengthscales, except near the grid size $h$ or the simulation domain size $L$. The structures must thus be scale invariant between $h$ and $L$, and the larger the ratio $L / h$ the better the approximation of a fully scaleinvariant structure.

In what follows we rescale pressures by $\mu_{r} U L_{x} / k$, lengths by $L_{x}$ and times by $L_{x} / U$. In the rescaled equations $\mu_{r}=1$ and the advancing fluid has viscosity $M=\mu_{a} / \mu_{r}$. All of the developments below are in terms of the new, rescaled variables.

\section{Method}

3.1 Numerical simulation of the Darcy equations with interfaces

The model equations above are solved using the methods described in Popinet $(2003,2009)$; Lagrée et al. (2011); Tryggvason et al. (2011), that is by discretizing the fields on an adaptive quadtree grid, using a projection method for the pressure, the time stepping and the incompressibility condition. The projection method results in an elliptic equation for the pressure, sometimes called a Poisson Equation, which is solved using the multigrid method described by Popinet (2003). It is the most time-consuming part of the whole procedure. The advection of the velocity fields is not necessary for the solution of the Darcy equations. We use the relaxation method described in Afkhami and Renardy (2013) and Lagrée et al. (2014) to approximate the solution of the Darcy equation. The interface is tracked using a Volume of Fluid (VOF) method with a Mixed Youngs-Centered Scheme (Tryggvason et al., 2011) for the determination of the normal vector and a Lagrangian-Explicit scheme for VOF advection. Viscosity is computed from the VOF fraction $C$ by an arithmetic mean. This arithmetic mean is followed by three steps of iteration of an elementary filtering. This whole set of methods is programmed either in the GERRIS flow solver (Popinet, 2014), or in the gerris scripts that were designed to launch these computations.

\subsection{Measurement of the fractal dimension}

\subsubsection{Box-counting method}

In the box-counting method, one determines the number $N(\ell)$ of boxes of size $\ell$ necessary to cover the entire object. Scaling is obtained if $N(\ell) \sim\left(L_{0} / \ell\right)^{D_{F}}$ (Feder, 1988). Comments on the results of this scaling are given in the next Section.

\subsubsection{Correlation or spatial-density decay method}

In the correlation dimension method, the probability $p(r)$ of finding pairs $\mathbf{x}, \mathbf{x}^{\prime}$ of points in the fractal separated by less than a distance $r$ is estimated by counting all the pairs of points in a set of points chosen at random in the fractal (Grassberger and Procaccia, 1983). Then the so-called Grassberger-Procaccia or correlation dimension is $p(r) \sim r^{D_{F}}$. In objects growing from a center, the probability of finding a point at the center is 1 , while the probability of finding a point away from the center is proportional to the integral of the density $d(r)$. Praud and Swinney (2005) thus measure the fractal dimension in Saffman-Taylor fingering with injection at the center using $d(r) \sim r^{D_{F}-2}$. When considering lateral injection as in our case instead of injection from a center, the same reasoning shows that the density $d(x)$ varies as $d(x) \sim x^{D_{F}-2}$. 


\subsubsection{Time-density decay or tip-point method}

The analysis of the average density of the fractal is correlated with its dimension as we show below. We consider a lateral injection with a constant, unity flux from the side. We then define the "average density" $C(t)$ of the fingering pattern as follows. Consider the most advanced point of the invading fluid, noted $X(t)$. The actual area of the invading fluid is $A(t)$ and since the invading fluid is fed into the domain at a constant rate then $A(t)=t L_{y}=t$. (Recall that lengths were rescaled so $L_{x}=L_{y}=1$ ). The rectangular slice of the domain between $x=0$ and $X(t)$ has area $X(t)$. Then we define the average density as the ratio of areas

$$
C(t)=A(t) / X(t)=t / X(t)
$$

To predict the behavior of $C(t)$, the fractal cluster can be seen as a collection of dendrites put one alongside the other in the $y$ direction of the computational domain. Each dendrite $i$ is included in a rectangle of size $\lambda_{i}(t) \times X_{i}(t)$, where $X_{i}(t)$ is the abcissa of the most advanced position and $\lambda_{i}(t)$ is the vertical extend of the dendrite. As the pattern evolves, and as observed above, some dendrites tend to dominate and inhibit the growth of the smaller dendrites. so that the number of active dendrites decreases and the length $\lambda_{i}(t)$ increases. We only consider the case where there is still more than one dendrite so $\lambda_{i}(t)<1$.

Since the velocity entering the domain has been rescaled to 1 , the area of a specific dendrite is $A_{r}=t \lambda_{i}(t)$. As the aspect ratio of the dendrite does not diverge, both dimensions of the rectangle $X_{i}(t) \sim \lambda_{i}(t)$ are of the same order and represent the large scale of the fractal. Moreover although each dendrite has a different extent their sizes are all of the same order so that $X_{i}(t) \sim X(t)$. From the usual box-counting method, one has $A_{r}=\ell^{2}(X(t) / \ell)^{D_{F}}$, with $\ell<X(t)$ an arbitrary fixed scale. Thus we obtain $t X(t) \sim K(\ell) X(t)^{D_{F}}$ where $K(\ell)$ is a constant independent of $t$. Thus the variation in time of $X$ is

$$
X(t) \sim t^{1 /\left(D_{F}-1\right)}
$$

We thus obtain from equations (6) and (7) the scaling $C(t) \sim t^{1-1 /\left(D_{F}-1\right)}=t^{-\delta}$ with $\delta=$ $-1+1 /\left(D_{F}-1\right)=\left(2-D_{F}\right) /\left(D_{F}-1\right)$. The position of the tip, i.e. the most advanced point of the fingering pattern is given by $X(t) \sim t^{1 / D_{F}-1}$.

\section{Results}

\subsection{Results in square geometry}

The results of the first simulations, performed with the parameters in Table 1 are presented in Figure 2. Two different refinements are used together with adaptive mesh refinement, defining two different cases (see Table 1).

As expected from the stability theory above, the simulation shows an instability of the interface, leading to finger creation. The fingers themselves are unstable and branch into smaller fingers, so that a self-similar structure begins to develop. In time, some large branched structures ("dendrites" or "trees") tend to dominate and inhibit the growth of the smaller dendrites. This successive branching leads to the formation of a self-similar structure with branches at several scales, except the smallest scale $h$ of the numerical method. Moreover the structures of the two simulations are similar to each other as expected in the absence of a physical length scale. The only difference is that the smallest length scale $h$ is smaller in case 2 than in case 1, resulting in the observation of smaller structures in case 


\begin{tabular}{|c|c|c|}
\cline { 2 - 3 } \multicolumn{1}{c|}{} & Case 1 & Case 2 \\
\cline { 2 - 3 } \multicolumn{1}{c|}{} & Figure 2(a) & Figure 2(b) \\
\hline Domain size & \multicolumn{2}{|c|}{$1 \times 1$} \\
\hline Minimum size of a cell & $h_{1}=2^{-11}$ & $h_{2}=2^{-12}$ \\
\hline Viscosity ratio $M$ & \multicolumn{2}{|c|}{$10^{-3}$} \\
\hline Interface & \multicolumn{2}{|c|}{ Sharp } \\
\hline
\end{tabular}

Table 1 Parameters of the simulation in square geometry

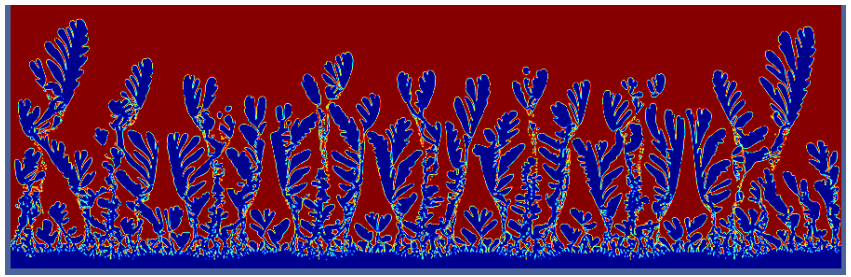

(a)

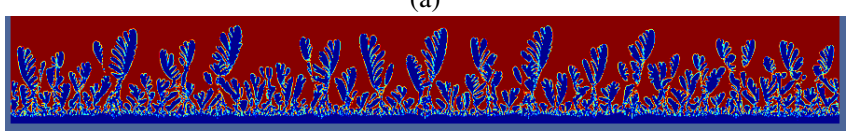

(b)

Fig. 2 Lateral injection of a less viscous fluid in a porous medium filled with a more viscous one for $M=10^{-3}$ (injection from the bottom): (a) case 1, (b) case 2.The characteristics of the simulations are presented in Table1. The CPU time is the same in both cases

2. As expressed above we expect self-similarity and fractality for length $h \ll \ell \ll L_{x}$. This can be verified in Figure 3 where we plot the measurement of the number of boxes $N(\ell)$ appearing in the box-counting method the fractal dimension in case 1 and in the experiment. For comparison, we show in Figure 3(b) the box counting results in the experimental case of Figure 1. In both cases we observe a remarkable power law scaling. The fractal dimension measured in the experimental case by box counting (Figure 3(b)) is $D_{F} \simeq 1.74$ close to the fractal dimension of Diffusion Limited Aggregation (DLA), $D_{F}=1.713 \pm 0.003$, see Davidovitch et al. (2000). DLA is known to correspond to the fingering process (Praud and Swinney, 2005). In the simulation case (Figure 3(a)) $D_{F} \simeq 1.92$ which is relatively far from the dimension of DLA. One explanation for that is that at the length scale corresponding to the thickness of the fingers $e$, the objects are not branched thin structures but fully two dimensional patches which result in a dimension of 2 . To observe self similarity one must investigate a range of scales $\ell \gg e$.

We also measure the fractal dimension by the correlation or density-decay method. The plot of $d(x)$ is on Figure 4 . We find $D_{F}=1.79$ in case 1 and $D_{F}=1.74$ in case 2 although the scaling does not work so well near the tip (the largest values of $x$ ) in case 2.

The instability inducing the fingering process is initialized by "numerical" noise (resulting from the imperfect convergence of the Poisson solver). This noise is axis-dependent, due to the oct-tree structure of the mesh (Popinet, 2003).

It is thus interesting to investigate the effect of a random heterogeneity in the domain by adding random perturbations of the local mobility $k / \mu$ (see Table 2 and Figure 5). This random perturbation has standard deviation

$$
\frac{\Delta(k / \mu)}{<k / \mu>}=5 \%
$$




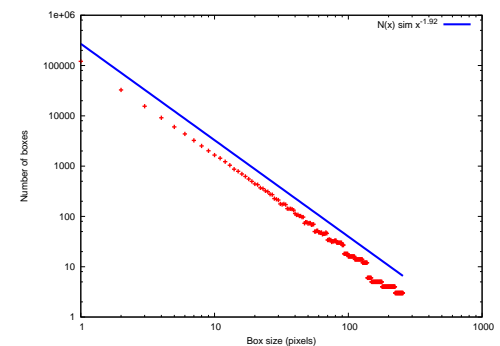

(a)

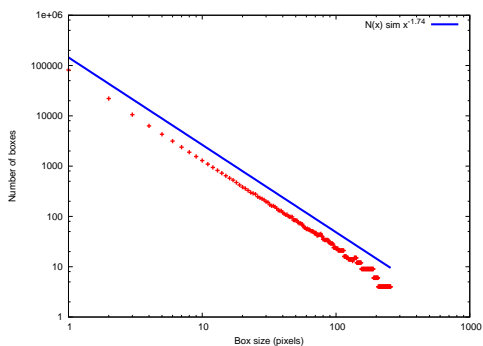

(b)

Fig. 3 A log-log plot of the number of boxes $N(\ell)$ versus the size $\ell$ of the boxes used in the box-counting algorithms (a) for simulation case 1, (b) for the experiment in Figure 1

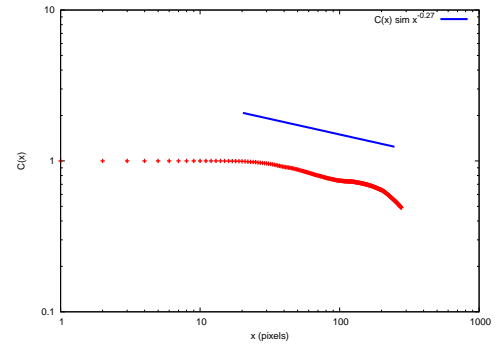

(a)

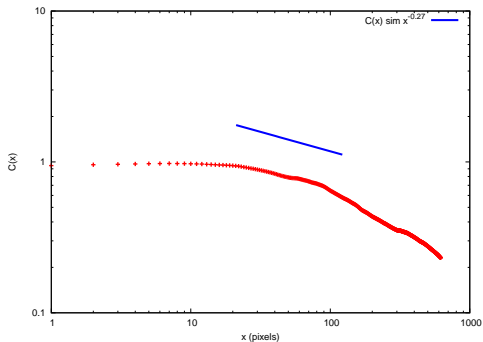

(b)

Fig. 4 A $\log -\log$ plot of the density $d(x)$ versus $x$ appearing in the correlation dimension algorithm (a) for simulation case 1, (b) for the experiment in Figure 1

\begin{tabular}{|c|c|c|}
\hline & Figure 5(a) & Figure 5(b) \\
\hline Domain size & \multicolumn{2}{|c|}{$1 \times 1$} \\
\hline Minimum size of a cell & \multicolumn{2}{|c|}{$h=2^{-10}$} \\
\hline Viscosity ratio $M$ & \multicolumn{2}{|c|}{$10^{-3}$} \\
\hline Interface & \multicolumn{2}{|c|}{ Sharp } \\
\hline Noise origin & "Mesh-induced" & Viscosity perturbation \\
\hline
\end{tabular}

Table 2 "Mesh-induced" noise (Lagrée et al., 2014) vs. perturbations of the local mobility $k / \mu$

The shape of the fingering patterns is clearly different. Without noise, the dendrites are more aligned with the $x$ axis, have less branching, and have smaller extent in the $y$ direction. This can be explained by the fact that noise promotes the branching of the dendrites. This may be related to a faster growth of the instability and a more intense splitting at the tip of the dendrite.

Since the advancing fingering patterns are not static objects characterized by a single fractal dimension but dynamic objects developping in time we introduce several "dynamic" measures of this growth process below. In Figure 6 we show the "average density" $C(t)$ of the fingering pattern. The connection of the average density with the fractal dimension was described in Section 3.2.3 above. As explained in that section a power-law scaling is expected for $C(t)$. Figure 6 shows indeed a power-law scaling after an initial regime where 


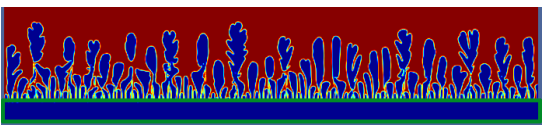

(a)

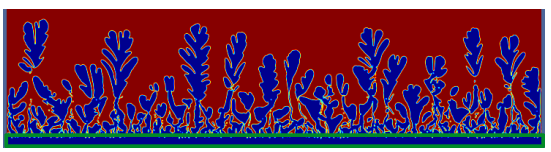

(b)

Fig. 5 Effect of the noise on the initiation of the fingering process (a) with uniform mobility. (b) with perturbations of the local mobility. The shape of the fingering patterns is clearly different. The characteristics of the simulations are presented in Table 2

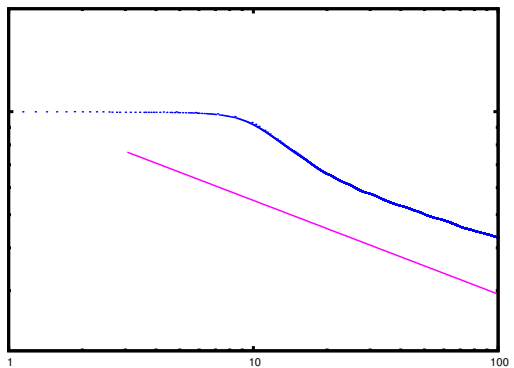

(a)

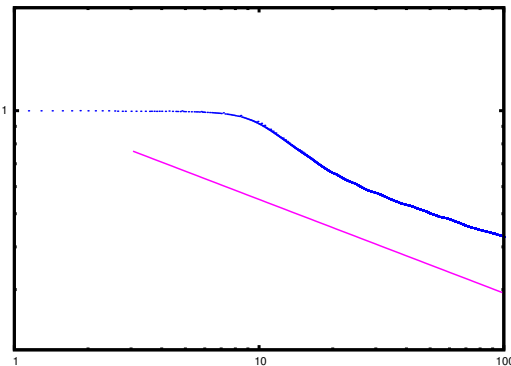

(b)

Fig. 6 The average density $C(t)$ of the fingering pattern versus time $t$ (see text). The fit $C(t) \sim t^{-\delta}$ is shown. (a) case $1(\delta=-0.27)$, (b) case $2(\delta=-0.31)$

$C(t)$ is constant. The constant $C(t)$ results from the initial condition in which the interface is flat, and is valid for the initial times before the instability has grown. The scaling is obtained with $\delta=0.27$ in case 1 and 0.31 in case 2 yielding respectively the time decay estimate $D_{F}=1.79$ and $D_{F}=1.76$. These values are close to, but not identical to the values obtained above by the box counting method applied directly to the images. Recall that with the box counting method we found $D_{F}=1.92$. It can be of interest to remark that the time decay values of $D_{F}$ are quite close to the fractal dimension of the DLA.

This was of course expected but has not been shown previously for lateral injection. Moreover the fact that the fractal dimension of the experiment is also close to that observed in DLA is an a posteriori validation of our simplified model.

Another characteristic of the development of branched patterns in time is the relation between the area of the invading fluid and the length of the interface. This relation has been shown numerically in Lagrée et al. (2014); Fast and Shelley (2006); Li et al. (2007) to be approximately a power law. For the simulations in this paper, the scaling is shown in Figure 7, with the following results: in case $1,\left(\alpha_{1}, \alpha_{\infty}\right)=(0.54 \pm 0.01,0.80 \pm 0.004)$, while $\left(\alpha_{1}, \alpha_{\infty}\right)=(0.38 \pm 0.005,0.76 \pm 0.01)$ in case 2 . The exponent $\alpha_{\infty}$ is close to the one observed in Lagrée et al. (2014); Fast and Shelley (2006); Li et al. (2007).

\subsection{Rectangular geometry}

It can be observed that fingers in the simulated patterns as well as in experiments are elongated in the direction of the flow. In order to observe a single branched finger with the widest possible range of scales we chose to reshape the computational domain to a rectangular domain of size $1 \times 1 / 4$. 


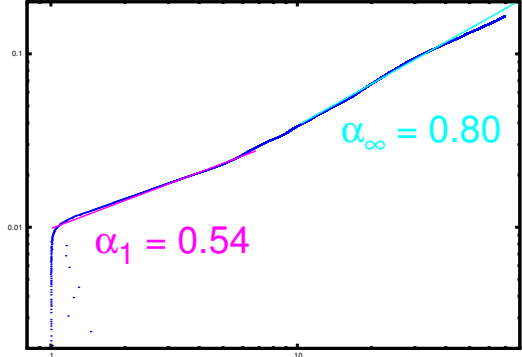

(a)

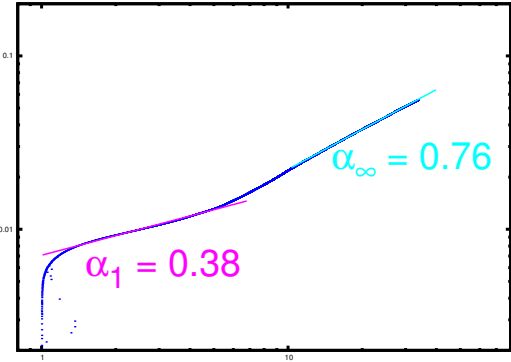

(b)

Fig. 7 Variation of the dimensionless area of the fingering process with respect to the dimensionless length of its interface: (a) case 1 , (b) case 2

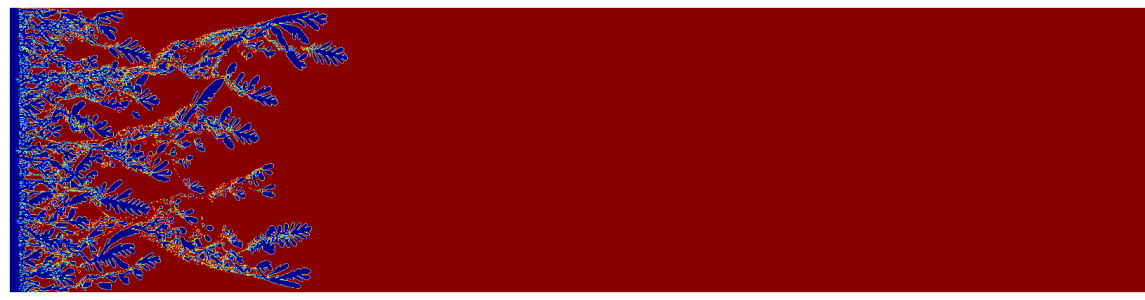

(a)

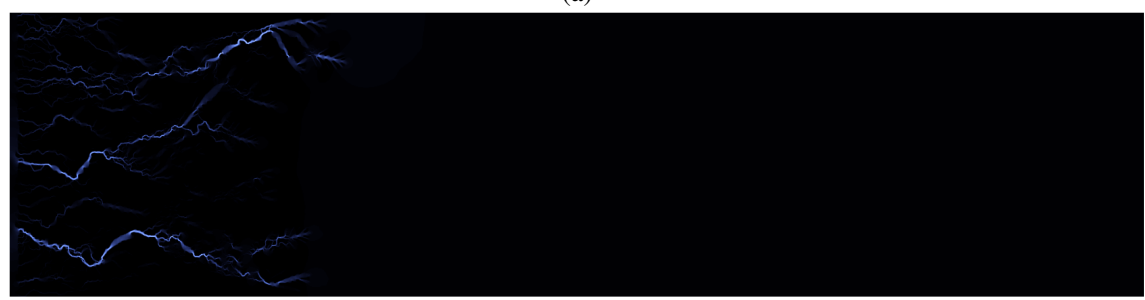

(b)

Fig. 8 Injection of a less viscous fluid in a rectangular porous medium with $M=10^{-3}$ (injection from the left-hand side): (a) the tracer (i.e. a marker-function such that the lower-velocity fluid is represented by one color and the higher-viscosity one by another), (b) the velocity field (closer to black: low velocity; closer to white: higher velocity)

The boundary conditions are the same as in subsection 4.1 The simulation is realized with the same parameters as case 2 above. The result is shown in Figure 8. The fractal dimension is measured by the correlation dimension method. We find $D_{F}=1.74 \pm 0.02$. One can observe the formation of relatively thick water channels, as observed experimentally by Skauge et al. (2012) (see the velocity field in Figure 8(b)).

In order to save computational time the simulation was repeated with a reduced refinement so the parameters are now those of case 1 above. The simulation is then continued until breakthrough time and the result is shown in Figure 9 The thick water channels are still present.

These simulations reproduce the fact that the post-breakthrough production rate is close to the pre-breakthrough one. The simulation yields that the post-breakthrough flux of the more-viscous fluid $q_{+}$is $q_{+} \approx 0.8 q_{-}$(with $q_{-}$the pre-breakthrough flux) at times close to breakthrough time in our simulations, as opposed to $q_{+} \sim 0.6 q_{-}$in Skauge et al. (2012) (the 


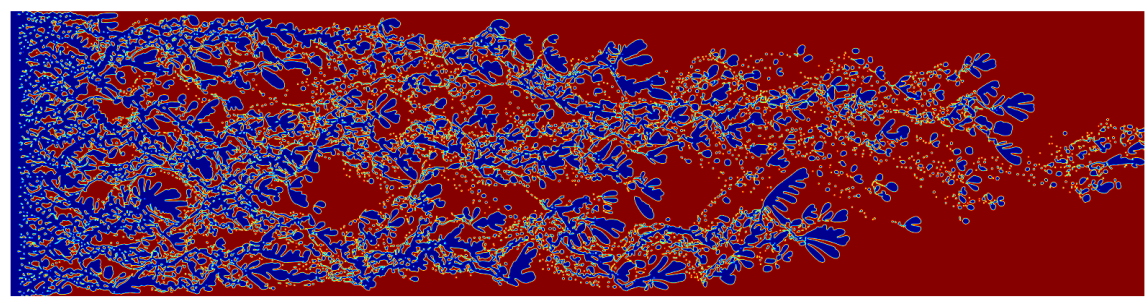

(a)

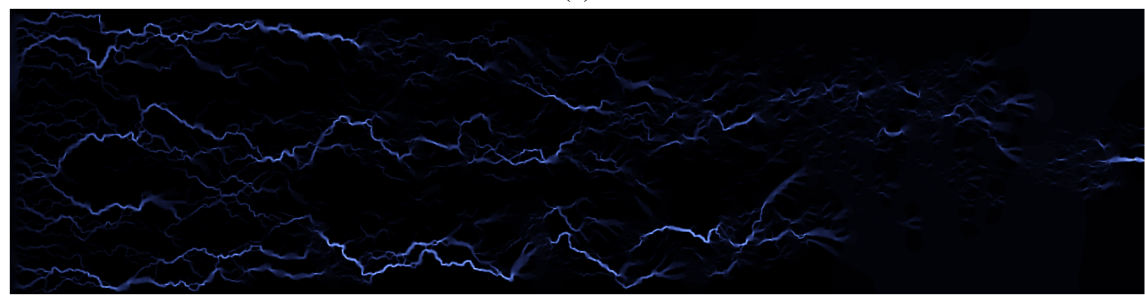

(b)

Fig. 9 Injection of a less viscous fluid in a rectangular porous medium till breakthrough with $M=10^{-3}$ (injection from the left-hand side): (a) the tracer, (b) the velocity field (closer to black: low velocity; closer to white: higher velocity)

experimental coefficient is only an approximate one, due to a lack of precision of the data we obtained from the curve). Consequently, as recovery continues after breakthrough, the pressure drop accross the domain after breakthrough is of the same order of magnitude as before breakthrough: The ration of pressure drops is $(\Delta p)_{+} /(\Delta p)_{-}=0.53$.

Both our results and those of Skauge et al. (2012) contradict the conventional view. Indeed, according to this view for sharp interfaces (i.e. when saturations are either 0 or 1), when injecting a less-viscous fluid with $\mu_{\text {injected }}=M \ll 1$ at a constant massflux, the pressure difference between the injection and exit sides is expected to decrease continuously towards 0 . For a viscous finger of width $w$ and length $L$, one can expect the post-breakthrough pressure difference $\Delta p_{+}$between the injection and exit faces to be of order $(w / L)^{-1} M \times \Delta p_{-}$, i.e. for $M=10^{-3}$ and $(w / L)^{-1}=10, \Delta p$ is expected to be divided by 100 . The divergence between our results and the conventional view is a result of the pinching process and the creation of several disconnected bubbles (see Figure 10). The origin of these bubbles, also seen in simulations of fingering with central injection by Lagrée et al. (2014) is not yet well understood. Indeed in the limit where $M=0$ it may be shown using the maximum principle that the interface should never recoil into the advancing fluid region (Lagrée et al., 2014). Thus the formation of bubbles is numerical or an effect of non-zero $M$.

The proportion of lower-viscosity phase was also obtained at several abscissae for different dimensionless times. The results are presented in Figure 11 for a viscosity ratio $M=1 / 2500$. These results are not consistent with those obtained in real quasi-2D experiments. In the experiments, the proporition of low viscosity phase at each abcissa is much lower than in simulations. This happens for the same reasons as presented above. The 3D aspect of the experiments introduces fingering in the third dimension, and the fronts in a real medium have regions of mixed phases behind and ahead of them.

The proportion of lower-viscosity phase at several abscissae for different times was compared for two different viscosity ratios: $M=1 / 2500$ and $M=1 / 7000$. The results are shown in Figure 12. In order to enhance the readability of the plots, the profiles at times $t_{3}$ and $t_{5}$ 


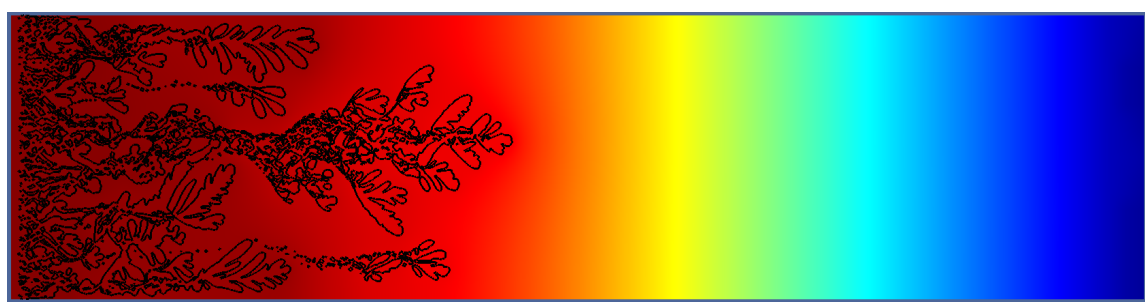

(a)

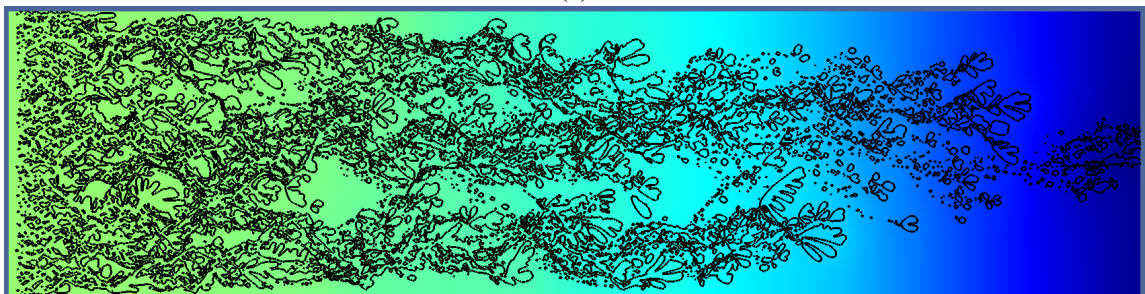

(b)

Fig. 10 Pressure field during the injection of a less viscous fluid in a rectangular porous medium with $M=$ $10^{-3}$ (injection from the left-hand side): (a) before breakthrough, (b) after breakthrough. The color scale is identical in both pictures

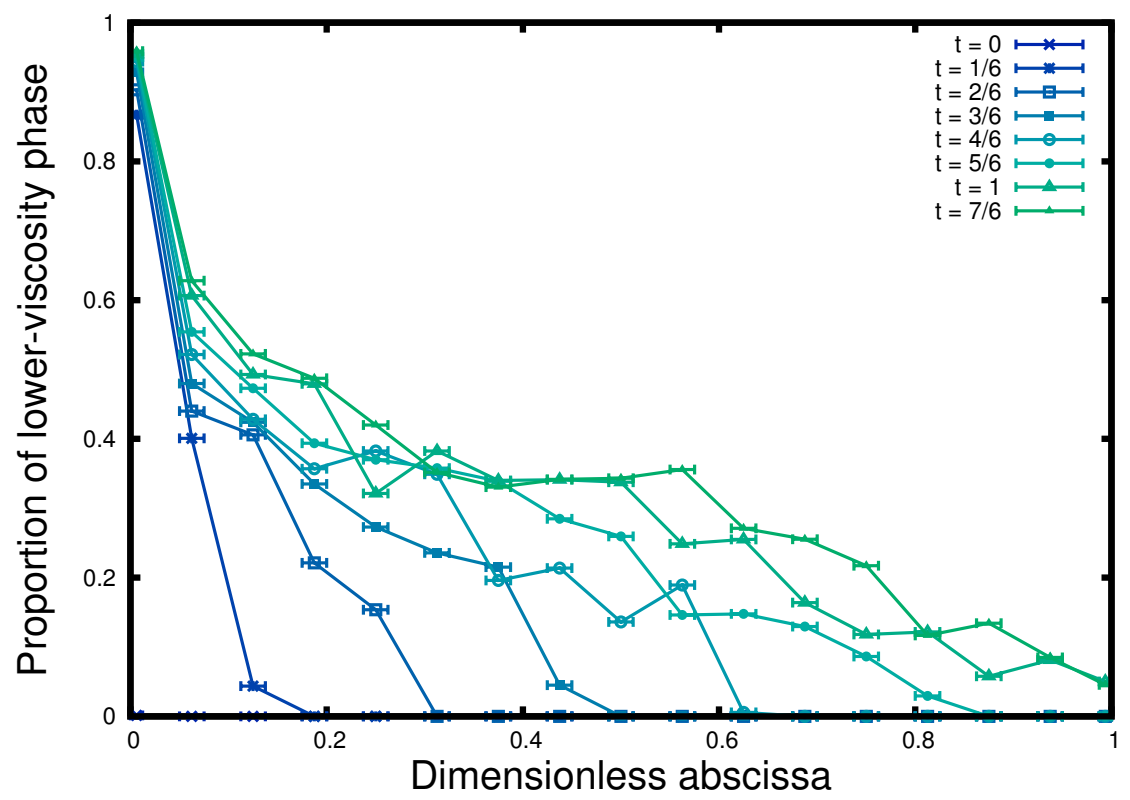

Fig. 11 Proportion of low-viscosity phase as a function of dimensionless abscissa for several dimensionless times. The data are average values over thin slices of porous medium between two specific abscissae (corresponding to the boundaries of the error bars) 


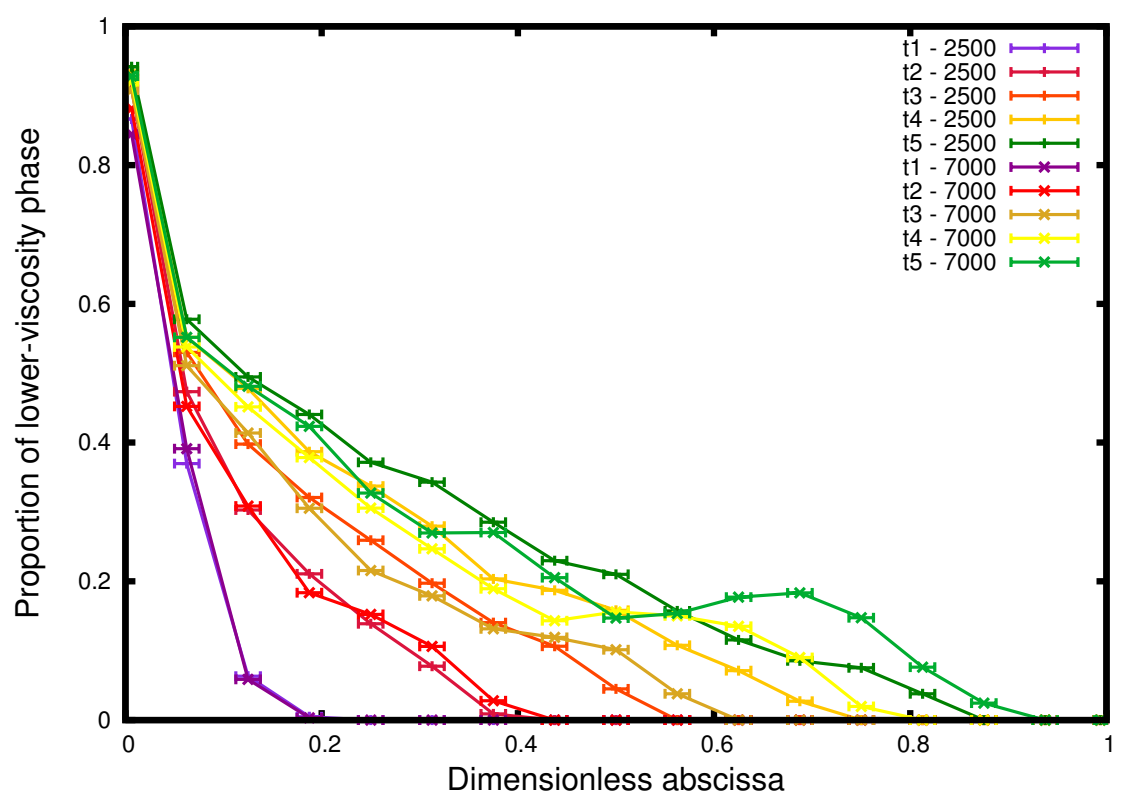

Fig. 12 Proportion of lower-viscosity phase as a function of abscissa for several times and different viscosity ratios $(M=1 / 2500$ and $M=1 / 7000)$. The data are average values over thin slices of porous medium between two specific abscissae (corresponding to the boundaries of the error bars)

were extracted from the series and replotted on Figure 13. The numerical results are consistent with the trend of the experimental results: indeed, for higher viscosity contrast the tip of the fingering process moves slightly more rapidly, ensuring breakthrough at shorter time. The expected recovery ratio at breakthrough is thus smaller for the case $M=1 / 7000$ than when $M=1 / 2500$, as was observed by Skauge et al. (2012). The difference between the dynamics for the two $M$ values is slight however.

It is worth discussing this result with reference to on one hand equation (7) which states that the advance of the tip depends only on the fractal dimension which in turn does not depend on $M$. On the other hand we have seen in figure 4 that the scaling does not work so well near the tip.

More generally the statistical properties of the fingering pattern, be they $D_{F}$ or the exponent $\alpha$, do not depend on $M$. Thus the observed dependence on $M$ is likely a second-order effect. The precise mechanisms involved remain to be investigated.

\subsection{Injection of an intermediate-viscosity fluid as a second step}

We finally consider the consecutive injection of both a low-viscosity fluid (hereafter called Fluid 1) and an intermediate-viscosity one (Fluid 2) in a porous medium originally filled with a high-viscosity fluid (Fluid 0 ). For this purpose, we focus on the same $1 \times 1 / 4$ domain as in the previous Section.

In a first step, we simulate the injection of the least viscous fluid in the domain similarly to what was presented beforehand. In their experiments, Skauge et al. (2012) waited till long after breakthrough before beginning the injection of the intermediate-viscosity fluid. In our 


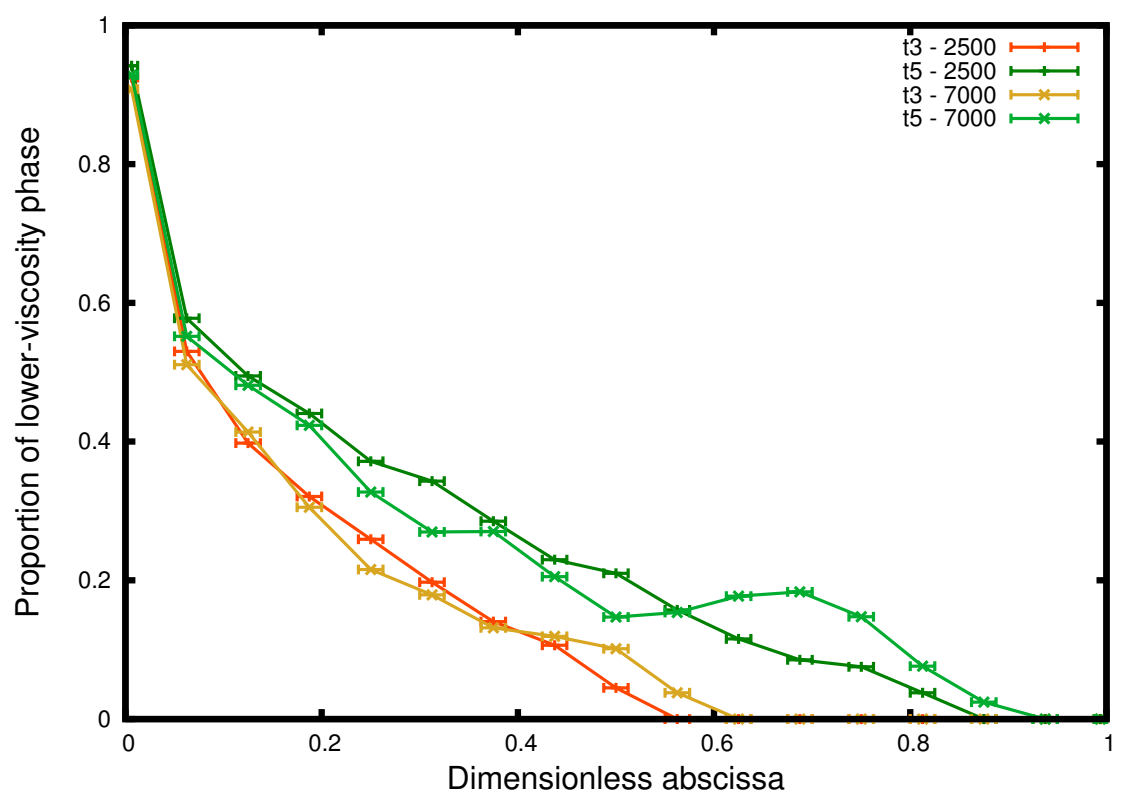

Fig. 13 Proportion of lower-viscosity phase as a function of abscissa for two specific times and different viscosity ratios $(M=1 / 2500$ and $M=1 / 7000)$. The data are average values over thin slices of porous medium between two specific abscissae (corresponding to the boundaries of the error bars)

simulations, we choose to begin this injection before breakthrough, in order to reduce the required CPU time.

The injection of Fluid 2 is realized from the same side as the one that was used to inject Fluid 1 . The different viscosity ratios are as follows

$$
\begin{aligned}
& M_{1}=\frac{\mu_{1}}{\mu_{0}}=\frac{1}{2500}, \\
& M_{2}=\frac{\mu_{2}}{\mu_{0}}=\frac{1}{50} .
\end{aligned}
$$

Three different cases are considered:

- case A: fluids 1 and 2 are not miscible. Mass conservation implies (Tryggvason et al., 2011), that the marker function $c$ varies as

$$
\partial_{t} c+\mathbf{u} \cdot \nabla c=0 .
$$

- case B: fluids 1 and 2 are miscible and diffuse one inside the other. The marker function thus obeys an advection-diffusion equation

$$
\partial_{t} c+\mathbf{u} \cdot \nabla c=D \Delta c
$$

with $D>0$. In this case, it was checked that $\tau_{\text {Diffusion }} \sim \tau_{\text {Viscosity }} \gg \tau_{\mathrm{CFL}}$.

- case C: This is a control case, destined to check the effects of numerical diffusion. To perform this control we discretize the same equation as in case B, with the same method, but with a zero coefficient of diffusion $(D=0)$. This checks the magnitude of the numerical diffusion of the advection scheme used to solve equation (12). The advection scheme is the Bell-Colella-Glaz second-order unsplit upwind scheme (Bell et al., 1989)). 


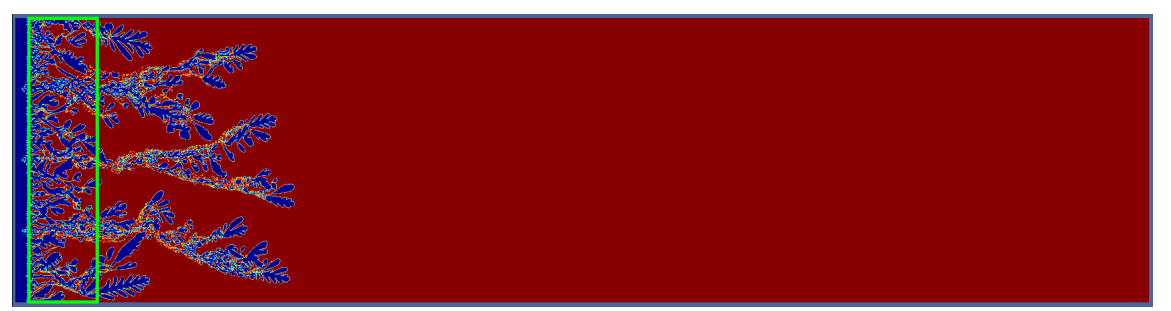

Fig. 14 Invasion of a porous medium by a single low-viscosity fluid. As no intermediate-viscosity fluid is injected, the high-viscosity fluid is not mobilized much inside the rectangle.

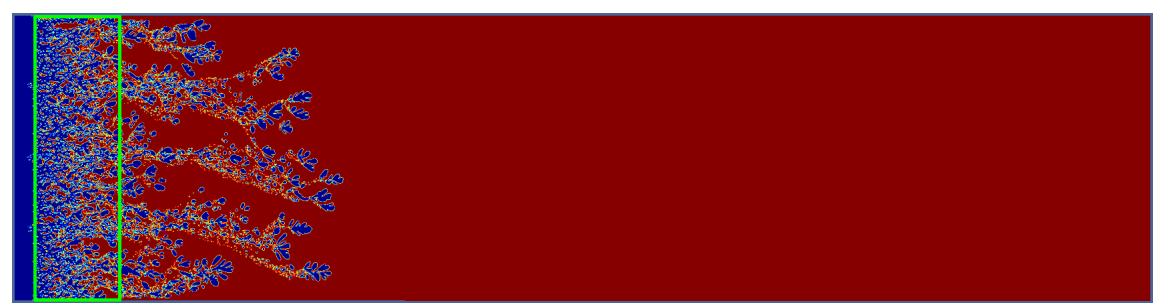

Fig. 15 Injection of an intermediate-viscosity fluid as a second step in a rectangular domain (injection from the left-hand side), case B. (with diffusion). In blue the phase containing both fluids 1 and 2 , in red fluid 0 . Inside the rectangle, fluid 0 is mobilized by fluid 2, compared to Figure 14 and thus the receding-fluid ratio decreases inside this slice of porous medium compared to the single-step case

In case $\mathrm{B}$, the interfaces between fluids 0 and 1 for one part, and fluids 0 and 2 for another part are still enforced to be sharp by the volume of fluid method. The interface between fluids 1 and 2 is the only diffuse one. To formulate the model differently, in cases B and C, there are two thermodynamic phases separated by a sharp interface. A tracer is dissolved in the invading phase, and "fluid 1" contains a zero concentration $c$ of the tracer while "fluid 2" contains a concentration $c=1$ of the same tracer. Case B is the closest to what actually occurs in the experiments presented by Skauge et al. (2012). We emphasize that sharp interfaces are in all cases a model. There is no change in the injection rate (constant massflux $\left.\mathbf{u}(-1 / 2, y)=\mathbf{e}_{\mathbf{x}}\right)$ when switching from Fluid 1 to Fluid 2 occurs. The side boundaries $y= \pm 1 / 2$ impose a slip condition, while the last side allows a "free" outflow condition for the fluids.

We perform simulations of these models with $D=0.11$ in dimensionless units. In all cases, the results reproduce what can be obtained experimentally. Indeed, the volume of Fluid 0 that remained inside the porous medium after the injection of Fluid 1 is mobilized behind the fingering front by the invading Fluid 2, as can be seen in Figure 15. The result obtained after invasion of only one low-viscosity fluid is recalled in Figure 14 to allow comparison. Indeed in Figures 17 and 18 it looks like the tracer is diffusing in both phases. The slight penetration of the tracer in the receding phase is however contained.

The mobilization of the receding fluid behind the fingering front is obvious when considering the proportion of Fluids 1 and 2 at a fixed dimensionless time for several values of $x$ (Figure 19). Indeed, for low $x$-values this proportion is increased by 10 to $20 \%$ for all cases A, B and C. compared to the simulation of the invasion of the porous medium by Fluid 1 only. On the contrary, the invasion by fluid 1 alone better fills higher abscissae for a fixed dimensionless time (especially abscissae 4 and 7 in the figure). In other words, injecting an intermediate-viscosity ratio as a second step leads to higher recovery ratios, i.e. the remain- 


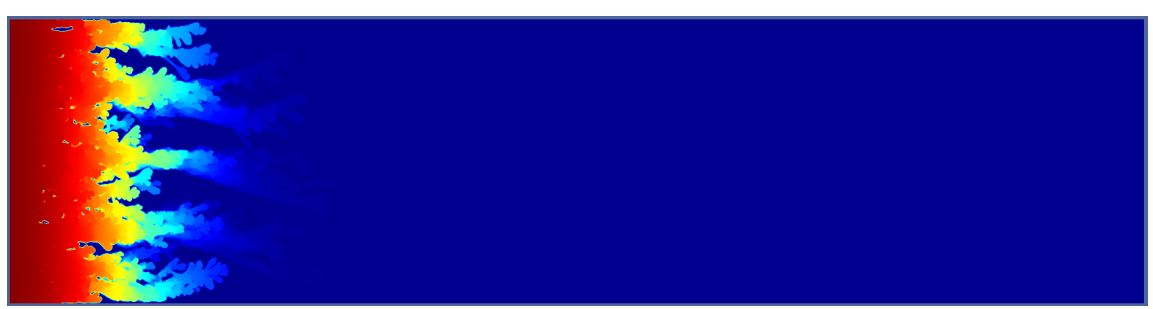

Fig. 16 Same simulation as in the previous Figure. In this figure, the receding fluid is in blue, and the mixture of advancing fluids is plotted with a color palette representing the mixture concentration $c$, varying from for $c=1$ (pure fluid 2) to blue for (pure fluid 1 or pure fluid 0 )

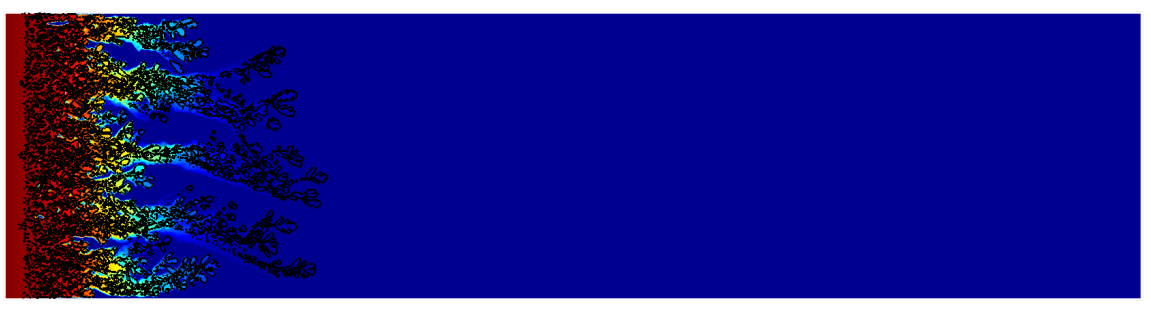

Fig. 17 Same simulation as in the previous Figure, with the same representation, but the position of the interface between the receding and the advancing fluid is shown as a black line

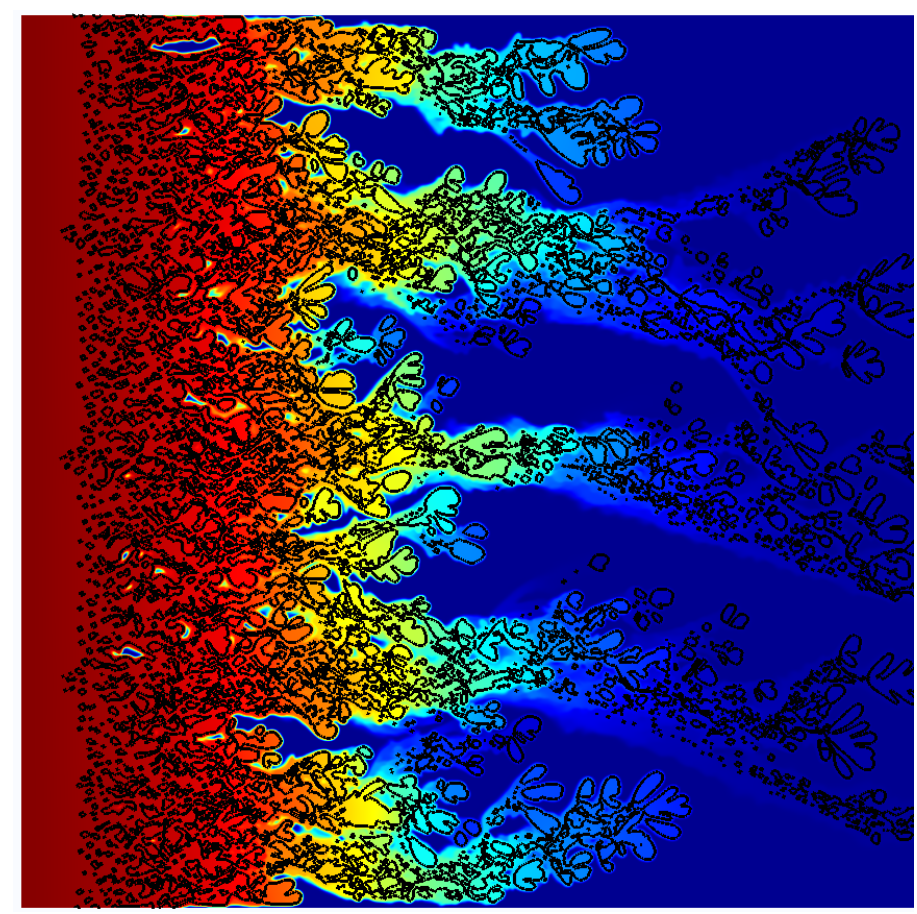

Fig. 18 A zoomed view of the previous Figure. It is seen that very little of the mixture has diffused accross the interface 


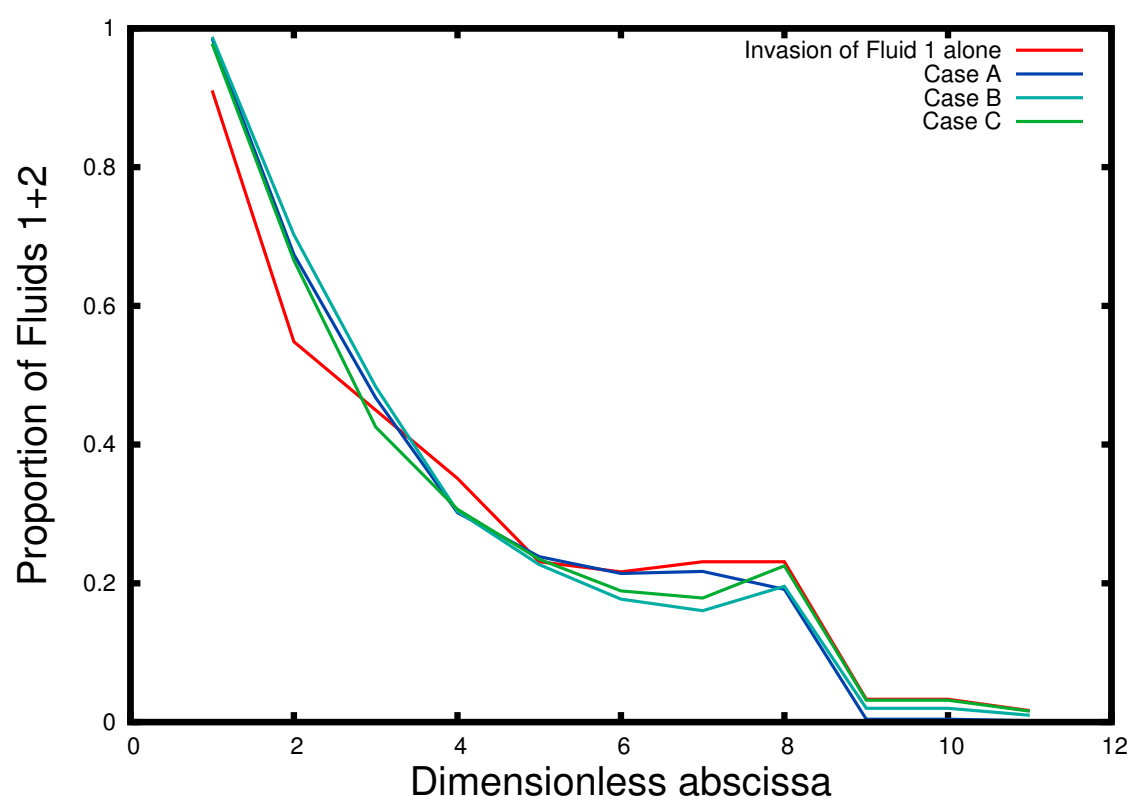

Fig. 19 Proportion of fluids 1 and 2 for several abscissae at a fixed injected volume. The data are average values over thin slices of porous medium between two specific abscissae

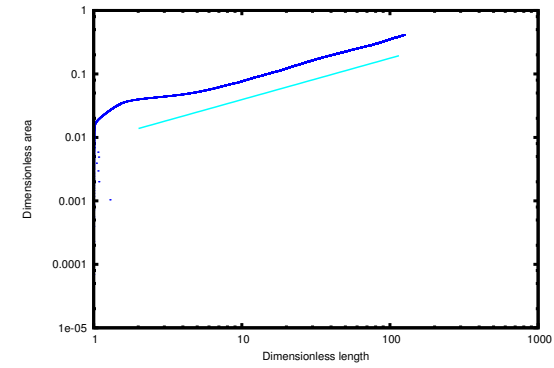

(a)

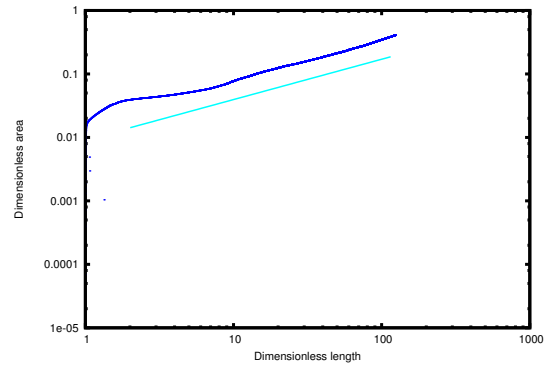

(b)

Fig. 20 Dimensionless area of the invading fluid as a function of the dimensionless length of the interface between invading and receding fluids: (a) case $\mathrm{A}\left(\alpha_{\infty}=0.64 \pm 0.01\right)$ and (b) case B $\left(\alpha_{\infty}=0.63 \pm 0.01\right)$

ing volume of Fluid 0 in the porous medium is lower after a double sweep by Fluid 1 then Fluid 2, than when injecting Fluid 1 alone.

Finally, the asymptotic coefficient $\alpha_{\infty}$ defined above is constant for the three cases $\left(\alpha_{\infty} \approx 0.63 \pm 0.02\right.$, see Figure 20$)$, but is significantly lower than when injecting Fluid 1 alone ( $\alpha_{\infty} \sim 0.8$, see Figure 7$)$. Consequently, when studying scaling properties of the twostep invasion of a porous medium by first a low-viscosity fluid and then an intermediateviscosity one, one can choose any of the three modelings studied above (for example the one presenting the best computational speed), with no lack of precision. This is once again due to the absence of a physical lengthscale in the problem. 


\section{Conclusion}

In this work, a sharp interface model has been used as a heuristic model for the simulation of viscous fingering in rectangular porous media with lateral injection. Both two- and threephase flows were investigated with a relatively large range of scales. The measured fractal dimensions are close to those of the DLA and consistent with what is previously known. The free solver Gerris was adapted for simulation of viscous fingering in rectangular porous media with lateral injection and two- and three-phase flows. The accuracy of the simulations was determined by comparing the computed fractal dimensions to the theoretical one of DLA. The use of a finer mesh creates a smaller lengthscale.

The method allows to simulate invasion of porous media until after breakthrough with interesting qualitative results. Indeed, the post-breakthrough rate at which the receding fluid is recovered remains close to the pre-breakthrough one. When performing simulations at two different viscosity ratios, the results are only slightly different, but the tip advance shows the same trend as in experiments.

Finally the comparison of two different models of the injection of an intermediateviscosity fluid in a second step shows no difference between the models. This shows that one can use either of them to study the scaling properties of such an injection. Indeed, the qualitative aspect is in all cases consistent with the experiments (the high-viscosity fluid is mobilized behind the fingering front).

Acknowledgements We thank TOTAL for the financial support and permission to publish this study.

\section{References}

S. Afkhami and Y. Renardy. A volume-of-fluid formulation for the study of co-flowing fluids governed by the Hele-Shaw equations. Phys. Fluids, 25(8):082001, 2013. doi: http://dx.doi.org/10.1063/1.4817374. URL http://scitation.aip.org/content/aip/journal/pof2/25/8/10.1063/1.4817374.

J. B. Bell, P. Colella, and H. M. Glaz. A second-order projection method for the incompressible navier-stokes equations. Journal of Computational Physics, 85(2):257 - 283, 1989. ISSN 0021-9991. doi: http://dx.doi.org/10.1016/0021-9991(89)90151-4. URL http://www.sciencedirect.com/science/article/pii/0021999189901514.

S. E. Buckley and M. C. Leverett. Mechanism of fluid displacement in sands. Transactions of the AIME, 146(1):107-116, 1942.

R. L. Chuoke, P. van Meurs, and C. van der Poel. The instability of slow, immiscible, viscous liquid-liquid displacements in permeable media. Trans. AIME, 216:188, 1959.

B. Davidovitch, A. Levermann, and I. Procaccia. Convergent calculation of the asymptotic dimension of diffusion limited aggregates: Scaling and renormalization of small clusters. Phys. Rev. E, 62:R5919-R5922, November 2000. doi: 10.1103/PhysRevE.62.R5919. URL http://link. aps .org/doi/10.1103/PhysRevE.62.R5919.

P. Fast and M. J. Shelley. Moore's law and the Saffman-Taylor instability. J. Comp. Phys., 212(1):1 - 5, 2006. ISSN 0021-9991. doi: 10.1016/j.jcp.2005.06.022. URL http://www.sciencedirect.com/science/article/pii/S0021999105003165.

J. Feder. Fractals. Plenum, 1988.

P. Grassberger and I. Procaccia. Characterization of Strange Attractors. Phys. Rev. Lett., 50:346-349, January 1983. doi: 10.1103/PhysRevLett.50.346. URL http://link.aps.org/doi/10.1103/PhysRevLett.50.346. 
H. S. Hele-Shaw. Flow of water. Nature, 58:520, 1898.

B. Lagrée, S. Zaleski, I. Bondino, C. Josserand, and S. Popinet. Scaling properties of viscous fingering. arXiv preprint, 2014.

P.-Y. Lagrée, L. Staron, and S. Popinet. The granular column collapse as a continuum: validity of a two-dimensional Navier-Stokes model with a $\mu(\mathrm{I})$-rheology. J. Fluid Mech., 686:378-408, 2011. ISSN 1469-7645. doi: 10.1017/jfm.2011.335.

S. Li, J. S. Lowengrub, and P. H. Leo. A rescaling scheme with application to the long-time simulation of viscous fingering in a Hele-Shaw cell. Journal of Computational Physics, 225(1):554 - 567, 2007. ISSN 0021-9991. doi: http://dx.doi.org/10.1016/j.jcp.2006.12.023. URL http://www.sciencedirect.com/science/article/pii/S0021999106006073.

K. J. Måløy, J. Feder, and T. Jøssang. Viscous Fingering Fractals in Porous Media. Phys. Rev. Lett., 55:2688-2691, December 1985. doi: 10.1103/PhysRevLett.55.2688. URL http://link.aps.org/doi/10.1103/PhysRevLett.55.2688.

S. Popinet. Gerris: a tree-based adaptive solver for the incompressible Euler equations in complex geometries. J. Comp. Phys., 190(2):572 - 600, 2003. ISSN 0021-9991. doi: http://dx.doi.org/10.1016/S0021-9991(03)00298-5.

S. Popinet. An accurate adaptive solver for surface-tension-driven interfacial flows. J. Comp. Phys., 228(16):5838 - 5866, 2009. ISSN 0021-9991. doi: http://dx.doi.org/10.1016/j.jcp.2009.04.042.

S. Popinet. Gerris flow solver-http://gfs.sourceforge.net/. 2014. URL http://gfs . sourceforge.net/.

O. Praud and H. L. Swinney. Fractal dimension and unscreened angles measured for radial viscous fingering. Phys. Rev. E, 72:011406, Jul 2005. doi: 10.1103/PhysRevE.72.011406. URL http://link. aps .org/doi/10.1103/PhysRevE.72.011406.

P. G. Saffman and G. I. Taylor. The penetration of a fluid into a porous medium or HeleShaw cell containing a more viscous fluid. P. R. S. London, 245(1242):312-329, 1958.

A. Skauge, P. A. Ormehaug, T. Gurholt, B. Vik, I. Bondino, and G. Hamon. 2-D Visualisation of Unstable Waterflood and Polymer Flood for Displacement of Heavy Oil. In SPE Improved Oil Recovery Symposium. Society of Petroleum Engineers, 2012.

G. Tryggvason, R. Scardovelli, and S. Zaleski. Direct Numerical Simulations of Gas-Liquid Multiphase Flows. Cambridge University Press, 2011. 